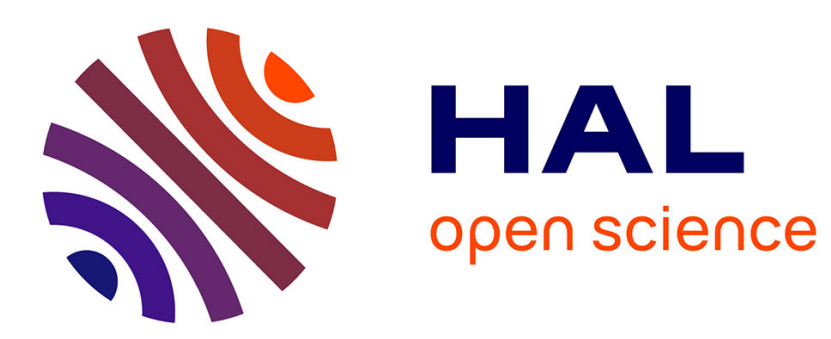

\title{
Inadéquation, hétérogénéité et contamination des échantillons soumis pour les datations radiocarbone
}

Georgette Delibrias, Pierre-Roland Giot

\section{To cite this version:}

Georgette Delibrias, Pierre-Roland Giot. Inadéquation, hétérogénéité et contamination des échantillons soumis pour les datations radiocarbone. Bulletin de la Société préhistorique française, 1970, 67 (5), pp.135-137. 10.3406/bspf.1970.4238 . hal-03402806

\section{HAL Id: hal-03402806 https://hal.science/hal-03402806}

Submitted on 25 Oct 2021

HAL is a multi-disciplinary open access archive for the deposit and dissemination of scientific research documents, whether they are published or not. The documents may come from teaching and research institutions in France or abroad, or from public or private research centers.
L'archive ouverte pluridisciplinaire HAL, est destinée au dépôt et à la diffusion de documents scientifiques de niveau recherche, publiés ou non, émanant des établissements d'enseignement et de recherche français ou étrangers, des laboratoires publics ou privés. 


\section{Inadéquation, hétérogénéité et contamination des échantillons} soumis pour les datations radiocarbone

Georgette Delibrias, P.-R. Giot

\section{Citer ce document / Cite this document :}

Delibrias Georgette, Giot P.-R. Inadéquation, hétérogénéité et contamination des échantillons soumis pour les datations radiocarbone. In: Bulletin de la Société préhistorique française. Comptes rendus des séances mensuelles, tome $67, n^{\circ} 5,1970$. pp. 135-137;

doi : https://doi.org/10.3406/bspf.1970.4238

https://www.persee.fr/doc/bspf_0249-7638_1970_num_67_5_4238

Fichier pdf généré le 08/09/2020 


\title{
Inadéquation, Hétérogénéité et Contamination des Echantillons
} soumis pour les Datations Radiocarbone

\author{
par (i. Delibrias et P.-R. Gio'T
}

La méthode de datation des matières carbonées d'origine organique par le dosage du ${ }^{14} \mathrm{C}$ est "majeure », elle a été imaginée il y a plus de 21 ans par W.-F. Libby (qui en fut récompensé tardivement par un prix Nobel de chimie en 1960 seulement), cela fait 18 ans que les premières séries consistantes de dates ont été publiées, cela fait environ 12 ans que la méthode a été suffisamment améliorée et diffusée pour donner lieu à une production de routine de nombreuses séries de dates, et cependant leur interprétation, leur exploitation par les « clients 》 des laboratoires de datation, laissent trop souvent à désirer. Pourtant les physiciens ont constamment mis en garde les utilisateurs contre les dangers de demander à la méthode plus qu'elle ne peut donner.

En comparant entre elles des dates radiocarbone on oublie trop souvent de vérifier si la différence sur laquelle on s'obnubile est statistiquement significative, on néglige le fait que le nombre exprimant le résultat d'une mesure d'âge est déduit de la mesure de l'activité de l’échantillon, laquelle est une grandeur aléatoire.

En comparant des dates radiocarbones et des dates historiques, on oublie que la vitesse du déroulement du temps du radiocarbone (sa " densité 》) n'a pas été constante, ou au contraire on s'exagère l'incidence des déviations possibles des âges radiocarbone par rapport aux âges vrais (qui, pour les temps post-glaciaires, semble devoir au maximum être inférieure à $13 \%$ de ceux-ci). Avec le progrès récent des méthodes de chronologies indépendantes (dendrochronologie, varves, thermoluminescence, géomagnétisme, données historiques) on doit espérer pour très bientôt des courbes ou tables de correction tout à fait satisfaisantes pour les der- niers 7500 ou même 10000 ans (1). Par contre la correspondance entre les âges apparents et les âges réels en période glaciaire reste du domaine de l'appréciation des plus spéculatives.

Devant une date absolue, il faut toujours observer une grande prudence, non pas parce que les mesures sont inexactes, mais parce que le matériel échantillonné pour la datation est souvent peu certain (2). On a pu écrire (3) : « one Carbon-14 date from a site or culture is no date », il faut des cohortes de dates qui s'étayent mutuellement (cependant il ne faut pas exagérer, une date unique faite sur un échantillon fiable est une indication bien utile). On a daté une matière organique qui est associée (avec un degré de probabilité plus ou moins

(1) V. Brcha ot E. Necstrexy. - Changes of the Earth's Magnetic Ficld and Radiocarbon Dating. Nature, 215, 1967, pp. 261-262. Changes of the Earth's Magnetic Field and Radiocarbon Dating. Pamalky Archeologicke, INHI-2, 1967, pp. j99-613.

P.E. DAyox, A. LoNi et D.C. (iREY - Fluctuation of Atmospheric $i$, 11 during the last Six Millenia. Journal of Geophysical Research, 71, 1966, pp. 10:5-1163.

C.IW. Fengl sox. - Bristlecone Pine : Science and Esthetics. Science, $159,1968, \mathrm{pp}$. 839-846.

E. Necstepsy - Absolute Chronology of the Neolithic and Aencolithic Periods in Central and Sout's Eastern Europe. Slonenska Archeologia, XVI-1, 1968, pp. 19-36. Absolute Chronolozy of the Neolithic and Aeneolithic priods in Central and South-East Europe II. Archeologicke rozhledy, XXI-6, 1969, pp. 78.3-810. A New Epoch in Radiocarbon Jating. Antiquity, XIIV 70, Pp. 38-45.

E.K. Ras.PH et M.C. Max. - Potential of thermoluminescence in supplementing radiocarbon dating. World Archeology, I, 1959, pp. $137-169$.

E.k. Raph et H.N. Micinat. - Problems of the Radiocarbon Calendar. Archaometry, 10, 1968, pp. 3-11.

H.E. Sicess. - Secular Variations of the Cosmic-Ray produced (arbon 11... .ournal of Geophysical Research, 70, 196.5, pn. 5937-5952. Bristlecone Pine Calibration of the Radiocarbon Time Scale from 1100 BC: to 1500 BC.. Radiocarbon Dating and belhods of low-Level Counling, Wien, 1967, pp. 143-151.

(2) J.C. Voger.. - Current Anthropolog!, VII, 1966, pp. 46-47. Ilelinium, IX: 1969, pp. 19-27.

(3) Н.Т. Waтенвок. - Antiquity, XXXIV, 1960, p. 18. 
grand) avec les objets ou les événements à dater, et association ne veut pas dire contemporanéité.

Beaucoup plus souvent qu'on ne l'imagine ou qu'on ne veut l'avouer, un résultat aberrant provient tout simplement du fait que l'association de l'échantillon daté et de l'événement que l'on en pense contemporain résulte d'une erreur d'interprétation ou de conditions de gisement incertaines. Les couches archéologiques sont loin d'être toutes idéalement scellées, et les causes de remaniements inaperçus sont nombreuses et variées. En particulier, les matériaux terreux ont subi, au fil des siècles, sur une échelle insoupçonnée, l'action des vers de terre, des insectes et des petits mammifères fouisseurs, sans compter celle des racines. De même des remplissages lâches, des pierrailles plus ou moins mêlées de terres, sont en fait des réseaux souterrains propices à des circulations intensives. Nous obtenons souvent des datations, non point à partir d'un gros morceau de charbon de bois unique, mais à partir d'une collection de petits grains de charbons de bois recueillis dispersés sur une surface ou dans un volume d'étendue variable. Il suffit d'un grain plus récent et donc de radioactivité notablement plus élevée pour rajjeunir considérablement le résultat : on obtient une sorte de moyenne proportionnelle entre deux âges, qu'on est en général dans l'incapacité de discriminer. En fait, un échantillon apparemment homogène peut être en réalité un mélange de grains de charbon de bois d'âges fort disparates. Il est cependant exceptionnel (quoique des remaniements de foyers anciens soient concevables et se produisent) que des grains plus anciens vieillissent une date ; pratiquement on n'a guère que des cas de rajeunissement, et si l'on dispose d'échantillons très abondants, on peut espérer, par des déterminations multiples, approcher de la réalité en tombant, dans ce jeu de «quitte ou double », sur un mélange aussi peu hétérogène que possible (mais ce jeu serait vite financièrement inacceptable).

Si l'on veut bien considérer l'expérience de l'un d'entre nous (P.-R. G.) sur les dates de son domaine géographique propre (4), formant une statistique d'environ 200 déterminations d'âge, on y trouvera :

- $2 \%$ de cas d'interprétation très difficile, c'est-à-dire des dates beaucoup plus anciennes que prévues, où le recours à une tentative d'explication par l'emploi de bois fossile de tourbières a été envisagé comme la seule porte de sortie ;

- $22 \%$ de cas qui ont conduit à une réinterprétation des conditions de gisement, cadrant parfaitement avec les faits et les expliquant

(4) P.R. (irot. - Chronique des datations radiocarbones armoricaines. Annales de Bretag!ne, LXVII, 1960, pp. 33-44 I.XVIII, 1961, pp. 21-24 ; IXIX, 1962, pp. 29-35; ;.XX, 1963, pp. 93-95 ; LXXII, 1965, pp. 133-147; ; IXXIII, 1966, pp. 124pp. 93-95 ; LXXII, 1965, pp. 133-147 ; IXXIII, 1966, pp. 124LXXVं, 1969, pp. 153-162. mieux que l'hypothèse de travail suivie au départ parce que la plus simple ;

- $7 \%$ de cas où un abaissement modéré de l'âge prévisible doit correspondre à une certaine hétérogénéité ou à une pollution probable de l'échantillon, hypothèses parfaitement admissibles mais sans évidences constatées sur le terrain :

- $5 \%$ de cas où un abaissement drastique de l'âge reste parfaitement inexplicable.

Le total apparent des divergences par rapport aux prévisions est de $36 \%$, mais en fait, compte tenu du doute scientifique avec lequel les hypothèses de travail sont élaborées, les vraies divergences ou surprises ne dépassent pas $10 \%$.

On réservera le terme de contamination aux sources de rajeunissement des échantillons homogènes (exceptionnellement de vieillissement) par absorption ou adsorption purement physique (c'est tout ce qui peut arriver à un charbon de bois, qui est chimiquement inerte) ou par action chimique. Un échantillon peut s'imprégner de substances carbonées étrangères, les radicelles, le mycélium des moisissures, les solutions d'acides humiques et de lignine provenant de sols sus-jacents étant les plus fréquemment invoqués, ou de carbonates provenant de milieux calcaires. C'est l'objet des pré-traitements de les enlever, avec une efficacité qui peut être presque totale ; les cas très rares où des pré-traitements ne sont pas effectués se produisent dans le cas d'échantillons insuffisants en poids, et où le «client » veut quand même absolument qu'on tente une datation (à ses risques et périls, et qu'en bonne logique on devrait lui refuser). Si l'échantillon n'est pas chimiquement inerte, et s'il est entré en réaction chimique avec les agents de contamination, il n'y a pas de remède. Une contamination de l'ordre de $1 \%$ en poids de Carbone récent est fréquente. Plus la substance carbonée est divisée, plus elle offre de surface à l'adsorption, plus le pré-traitement sera difficile. Aussi les gros morceaux de bois bien conservés et les gros morceaux de charbon de bois (au sens strict de bois carbonisé) donnent les meilleurs résultats, c'est-à-dire les plus vieux (2). Le bois et le charbon peuvent plus rigoureusement être traités par des produits chimiques pour éliminer toutes traces de substances humiques étrangères. Dans le cas de la tourbe et de ce qui est nommé «os brûlé 》 et «cendres »c'est rarement le cas (2). Des résultats encourageants ont été obtenus à partir de l'os non brûlé (par extraction du résidu de collagène). Les radicelles forment facilement 1 à $2 \%$ du poids en Carbone récent d'un échantillon qu'elles ont contaminé ; elles ne peuvent être dissoutes par les agents chimiques de pré-traitement, leur extraction se fait mécaniquement dans la mesure où on les voit.

En fait ces processus de contamination sont peu sensibles pour les échantillons protohistoriques ou des temps néolithiques. Ils prennent 
vite une incidence drastique pour des échantillons paléolithiques. Le tableau suivant montre les diminutions d'âge pour 4 niveaux de pollution pour des échantillons dont on indique l'âge qui aurait dû être obtenu :

\begin{tabular}{|c|c|c|c|}
\hline & $\begin{array}{l}10000 \\
\pm 200\end{array}$ & $\begin{array}{l}20000 \\
\pm 600\end{array}$ & $\begin{array}{c}30000 \\
\pm \quad 1800\end{array}$ \\
\hline 0,1 ? & $50^{\star}$ & $80^{*}$ & $340^{\star}$ \\
\hline g & $170^{\star}$ & 860 & 2800 \\
\hline 0 & 950 & 3600 & 9200 \\
\hline $10 \%$ & 1700 & 6000 & 13200 \\
\hline
\end{tabular}

* se confond avec l'erreur statistique.

On obtient une répartition très dispersée vers les valeurs les plus jeunes pour différents échantillons d'un même ẩge, affectés par une pollution variable. Au fur et à mesure que les résultats s'accumulent, les dates peuvent se grouper auprès d'une certaine valeur maximale qui représentera alors l'âge véritable de l'événement en cours de datation.

Le deuxième tableau ci-après donne une idée des effets des mélanges ou des pollutions sur des échantillons jusqu'à 10000 ans, c'est-à-dire pour le Post-glaciaire. On verra qu'en pratique les effets d'une contamination proprement dite (au maximum de l'ordre de 2 à $3 \%$ de l'échantillon) sont plutôt négligeables en effet, et que lorsqu'on constate un écart appréciable entre l'âge obtenu et l'âge supposé, l'on doit beaucoup plus sérieusement invoquer la non-homogénéité de l'échantillon, c'est-à-dire son mélange avec une substance identique plus récente, ou son inadéquation pure et simple, bref le plus souvent mettre en cause les conditions de gisement. Qu'on veuille bien avoir le courage de le faire.

Dans ce tableau, les additions sont calculées comme étant en C actuel. Dans la pratique, les intrusions ou les contaminations sont ellesmêmes d'un certain âge (souvent étalé dans le temps), ce qui implique des pourcentages d'addition beaucoup plus élevés. Pour fixer les idées, si pour un échantillon néolithique qui devrait donner un âge de 5000 ans, et qu'on sait mélangé avec du charbon de bois de l'Age du Fer vieux de 2500 ans, on obtient un âge apparent de 4000 ans, c'est que le mélange est dans des proportions voisines de $36 \%$ de pollution contre $64 \%$ de charbon néolithique.

\begin{tabular}{|c|c|c|}
\hline $\begin{array}{l}\text { Age réel } \\
\text { ans }\end{array}$ & $\begin{array}{c}\text { Rajeunissement } \\
\text { ans }\end{array}$ & $\begin{array}{l}\text { Pollution en poids } \\
\text { par du C récent - \% }\end{array}$ \\
\hline 10000 & 1000 & 5,5 \\
\hline 10000 & 500 & 3 \\
\hline 10000 & 200 & $1,1^{\star}$ \\
\hline 8000 & 1000 & 8 \\
\hline 8000 & 500 & 3,7 \\
\hline 8000 & 200 & $1,4^{\star}$ \\
\hline 6000 & 1000 & 12 \\
\hline 6000 & 500 & 5,8 \\
\hline 6000 & 200 & 2,4 \\
\hline 6000 & 100 & 1,3 * \\
\hline 5000 & 1000 & 15,5 \\
\hline 5000 & 500 & 7,7 \\
\hline 5000 & 200 & 3 \\
\hline 5000 & 100 & $1,5^{\star}$ \\
\hline 4000 & 1000 & 20 \\
\hline 4000 & 500 & 10 \\
\hline 4000 & 200 & 4 \\
\hline 4000 & 100 & $2,2^{\star}$ \\
\hline 3000 & 1000 & 30 \\
\hline 3000 & 500 & 14 \\
\hline 3000 & 200 & 5,7 \\
\hline 3000 & 100 & $2,8^{\star}$ \\
\hline 2000 & 1000 & 47 \\
\hline 2000 & 500 & 23 \\
\hline 2000 & 200 & 8,1 \\
\hline 2000 & 100 & 4 * \\
\hline 1000 & 500 & 31 \\
\hline 1000 & 200 & 18,5 \\
\hline 1000 & 100 & 8 * \\
\hline
\end{tabular}

